\title{
A BOTT INVERTED MODEL FOR EQUIVARIANT UNITARY TOPOLOGICAL $K$-THEORY
}

\author{
MARKUS SPITZWECK and PAUL ARNE ØSTV ÆR
}

\begin{abstract}
Let $\mathrm{A}$ be an abelian compact Lie group. We show the Bott inverted suspension spectrum of the projective space that classifies A-equivariant line bundles is a model for A-equivariant unitary topological $K$-theory.
\end{abstract}

\section{Introduction}

Let $\mathrm{A}$ be an abelian compact Lie group. For $\alpha \in \operatorname{Hom}(\mathrm{A}, \mathrm{R} / \mathrm{Z})$ let $\mathrm{C}_{\alpha}$ denote the complex numbers with A-action $a . z=e^{2 \pi i \alpha(a)} z$. Then $\mathrm{C}_{\alpha} \otimes \mathrm{C}_{\alpha^{\prime}}=\mathrm{C}_{\alpha+\alpha^{\prime}}$ and

$$
\mathscr{U}_{\mathrm{A}} \equiv \bigoplus_{\alpha} \mathrm{C}_{\alpha} \otimes \mathrm{C}^{\infty}
$$

is a complete A-universe with a contractible space of linear isometries

$$
\mathscr{U}_{\mathrm{A}} \otimes \mathcal{U}_{\mathrm{A}} \rightarrow \mathscr{U}_{\mathrm{A}} .
$$

It follows that the projective space $\mathrm{P}\left(\mathscr{U}_{\mathrm{A}}\right)$ that classifies A-equivariant line bundles acquires a natural $\mathrm{A}$-action and also an abelian group structure up to equivariant homotopy. More generally, recall the $n$th Grassmannian $\mathrm{Gr}_{n}\left(\mathscr{U}_{\mathrm{A}}\right)$ is an A-space that classifies A-equivariant $n$-dimensional bundles. Denote the corresponding colimit by $\operatorname{Gr}\left(\mathscr{U}_{\mathrm{A}}\right)$. Then the composite map

$$
\mathrm{P}\left(\mathscr{U}_{\mathrm{A}}\right)=\mathrm{Gr}_{1}\left(\mathscr{U}_{\mathrm{A}}\right) \longrightarrow\{1\} \times \operatorname{Gr}\left(\mathscr{U}_{\mathrm{A}}\right) \longrightarrow \mathrm{Z} \times \operatorname{Gr}\left(\mathscr{U}_{\mathrm{A}}\right)
$$

induces, via the adjunction between equivariant spaces and spectra, a map

$$
\Sigma^{\infty} \mathrm{P}\left(\mathscr{U}_{\mathrm{A}}\right)_{+} \longrightarrow \mathrm{KU}_{\mathrm{A}}
$$

from the suspension spectrum of the projective space to the representing spectrum for A-equivariant unitary topological $K$-theory.

By inverting the Bott map $\beta$ for $\Sigma^{\infty} \mathrm{P}\left(\mathcal{U}_{\mathrm{A}}\right)_{+}$we show the following result.

Received 2 August 2008, in revised form 25 June 2009. 
THEOREM 1.1. There is an isomorphism

$$
\Sigma^{\infty} \mathrm{P}\left(\mathscr{U}_{\mathrm{A}}\right)_{+}\left[\beta^{-1}\right] \stackrel{\cong}{\longrightarrow} \mathrm{KU}_{\mathrm{A}}
$$

in the equivariant stable homotopy category.

The paper is layed out as follows. Section 2 recalls the definition of equivariant oriented cohomology theories. The main examples relevant to this paper are $\mathrm{MU}_{\mathrm{A}}$ and $\mathrm{KU}_{\mathrm{A}}$. Section 3 introduces Bott inverted $\Sigma^{\infty} \mathrm{P}\left(\mathscr{U}_{\mathrm{A}}\right)_{+}$and an $\mathrm{A}-$ equivariant orientation class. A proof of Theorem 1.1 follows by combining the A-equivariant Conner-Floyd theorem with the above. The details of the argument are recorded in Section 4.

For legibility we allow a uniform notation for "Bott elements and maps" trusting that the precise meaning will be clear from the context. The writing of this paper was inspired by Snaith's isomorphism for naive equivariant cohomology theories in [9] and the proof of an analogous motivic result given in [10].

\section{Equivariant oriented cohomology theories}

We refer to [4] for background on oriented cohomology theories and formal group laws in equivariant homotopy theory. Let $\mathrm{E}_{\mathrm{A}}^{*}(-)$ be an A-equivariant cohomology theory, $\varepsilon$ the trivial representation and $\alpha$ some 1-dimensional representation. There are inclusions

$$
*_{\varepsilon} \equiv \mathrm{P}(\varepsilon) \subseteq \mathrm{P}(\varepsilon \oplus \alpha) \subseteq \mathrm{P}\left(\mathcal{U}_{\mathrm{A}}\right) .
$$

An element of $\mathrm{E}_{\mathrm{A}}^{*}\left(\mathrm{P}\left(\mathscr{U}_{\mathrm{A}}\right), *_{\varepsilon}\right)$ is called an orientation class for $\mathrm{E}_{\mathrm{A}}^{*}(-)$ if for every $\alpha$ it restricts to a generator of

$$
\mathrm{E}_{\mathrm{A}}^{*}\left(\mathrm{P}(\varepsilon \oplus \alpha), *_{\varepsilon}\right)=\widetilde{\mathrm{E}}_{\mathrm{A}}^{*}(\mathrm{P}(\varepsilon \oplus \alpha)) .
$$

The homotopical unitary cobordism spectrum $\mathrm{MU}_{\mathrm{A}}$ has a canonical orientation, and therefore there exists an equivariant formal group law over $\mathrm{MU}_{\mathrm{A}}^{*}[4$, $\S 13]$. Any degree 2 orientation class for $E_{A}^{*}(-)$ determines a unique orientation preserving ring map between $\mathrm{A}$-spectra $\mathrm{MU}_{\mathrm{A}} \rightarrow \mathrm{E}$ by [1, Theorem 1.2].

As in $[4, \S 10]$ one shows the A-equivariant unitary topological $K$-theory spectrum acquires a degree 2 orientation class

$$
\beta^{-1}(1-\mathrm{L}) \in \mathrm{KU}_{\mathrm{A}}^{2}\left(\mathrm{P}\left(\mathscr{U}_{\mathrm{A}}\right), *_{\varepsilon}\right) .
$$

Here $\mathrm{L}$ denotes the canonical line bundle over $\mathrm{P}\left(\mathscr{U}_{\mathrm{A}}\right)$. The corresponding coefficient ring $\operatorname{Rep}_{\mathrm{U}}(\mathrm{A})\left[\beta, \beta^{-1}\right.$ ] (that is, the Laurent polynomial ring in the variable $\beta$ over the representation ring) represents A-equivariant strictly multiplicative formal group laws [5]. 


\section{The Bott inverted equivariant infinite projective space}

Denote by $\varepsilon \oplus \varepsilon$ the trivial 2-dimensional representation. The identity map on $\mathrm{P}\left(\mathscr{U}_{\mathrm{A}}\right)$ defines classes

$$
\xi_{\infty} \in\left(\Sigma^{\infty} \mathrm{P}\left(\mathscr{U}_{\mathrm{A}}\right)_{+}\right)_{\mathrm{A}}^{0}\left(\mathrm{P}\left(\mathscr{U}_{\mathrm{A}}\right)\right), \quad \xi_{1} \equiv \xi_{\infty_{*_{\varepsilon} \oplus \varepsilon}} \in\left(\Sigma^{\infty} \mathrm{P}\left(\mathscr{U}_{\mathrm{A}}\right)_{+}\right)_{\mathrm{A}}^{0}(\mathrm{P}(\varepsilon \oplus \varepsilon)) .
$$

Clearly $\xi_{\infty}$ pulls back to the unit element of $\left(\Sigma^{\infty} \mathrm{P}\left(\mathscr{U}_{\mathrm{A}}\right)_{+}\right)_{\mathrm{A}}^{0}$ and the class $1-\xi_{1}$ is sent to $1-\mathrm{L}_{\text {in }} \mathrm{KU}_{\mathrm{A}}^{0}(\mathrm{P}(\varepsilon \oplus \varepsilon))$ under the naturally induced map

$$
\left(\Sigma^{\infty} \mathrm{P}\left(\mathscr{U}_{\mathrm{A}}\right)_{+}\right)_{\mathrm{A}}^{0}(\mathrm{P}(\varepsilon \oplus \varepsilon)) \longrightarrow \mathrm{KU}_{\mathrm{A}}^{0}(\mathrm{P}(\varepsilon \oplus \varepsilon)) .
$$

We may also consider the image of the reduced cohomology class $1-\xi_{1}$ in the coefficient ring of $\mathrm{P}\left(\mathscr{U}_{\mathrm{A}}\right)$ by virtue of the identification

$$
\left(\Sigma^{\infty \mathrm{P}\left(\mathscr{U}_{\mathrm{A}}\right)_{+}}\right)_{\mathrm{A}}^{0}(\mathrm{P}(\varepsilon \oplus \varepsilon))=\left(\Sigma^{\infty} \mathrm{P}\left(\mathscr{U}_{\mathrm{A}}\right)_{+}\right)_{2} .
$$

(Stably we have $\mathrm{P}(\varepsilon \oplus \varepsilon)_{+}=S^{0} \vee S^{2}$.) There exists a corresponding map

$$
\Sigma^{\infty} \mathrm{P}(\varepsilon \oplus \varepsilon) \longrightarrow \Sigma^{\infty} \mathrm{P}\left(\mathcal{U}_{\mathrm{A}}\right)_{+}
$$

and taking the adjoint of

$\Sigma^{\infty} \mathrm{P}(\varepsilon \oplus \varepsilon) \wedge \Sigma^{\infty} \mathrm{P}\left(\mathscr{U}_{\mathrm{A}}\right)_{+} \longrightarrow \Sigma^{\infty} \mathrm{P}\left(\mathscr{U}_{\mathrm{A}}\right)_{+} \wedge \Sigma^{\infty} \mathrm{P}\left(\mathscr{U}_{\mathrm{A}}\right)_{+} \longrightarrow \Sigma^{\infty} \mathrm{P}\left(\mathscr{U}_{\mathrm{A}}\right)_{+}$ yields the Bott map

$$
\beta: \Sigma^{\infty} \mathrm{P}\left(\mathscr{U}_{\mathrm{A}}\right)_{+} \longrightarrow \Sigma^{-2} \Sigma^{\infty} \mathrm{P}\left(\mathscr{U}_{\mathrm{A}}\right)_{+} .
$$

An inspection reveals that $\beta$ coincides with the Bott map introduced in [9].

Definition 3.1. The Bott inverted $\mathrm{P}\left(\mathscr{U}_{\mathrm{A}}\right)$ is the homotopy colimit

$\Sigma^{\infty} \mathrm{P}\left(\mathscr{U}_{\mathrm{A}}\right)_{+}\left[\beta^{-1}\right] \equiv \operatorname{hocolim}\left(\Sigma^{\infty} \mathrm{P}\left(\mathscr{U}_{\mathrm{A}}\right)_{+} \stackrel{\beta}{\longrightarrow} \Sigma^{-2} \Sigma^{\infty} \mathrm{P}\left(\mathscr{U}_{\mathrm{A}}\right)_{+} \stackrel{\Sigma^{-2} \beta}{\longrightarrow} \cdots\right)$.

We note there exists an evident ring structure on $\Sigma^{\infty} \mathrm{P}\left(\mathscr{U}_{\mathrm{A}}\right)_{+}\left[\beta^{-1}\right]$ and also an associated A-equivariant cohomology theory. Moreover, $\beta$ maps to the Bott element for $\mathrm{KU}_{\mathrm{A}}$ under $\left(\Sigma^{\infty} \mathrm{P}\left(\mathscr{U}_{\mathrm{A}}\right)_{+}\right)_{2} \rightarrow\left(\mathrm{KU}_{\mathrm{A}}\right)_{2}$ since the latter element corresponds to the virtual vector bundle $1-\mathrm{L}$ over $\mathrm{P}(\varepsilon \oplus \varepsilon)$. In what follows, we denote by the same letters the images of the classes $\beta$ and $\xi_{\infty}$ under maps induced by

$$
\Sigma^{\infty} \mathrm{P}\left(\mathscr{U}_{\mathrm{A}}\right)_{+} \longrightarrow \Sigma^{\infty} \mathrm{P}\left(\mathscr{U}_{\mathrm{A}}\right)_{+}\left[\beta^{-1}\right] .
$$


LEMMA 3.2. The class

$$
\beta^{-1}\left(1-\xi_{\infty}\right) \in\left(\Sigma^{\infty} \mathrm{P}\left(\mathscr{U}_{\mathrm{A}}\right)_{+}\left[\beta^{-1}\right]\right)_{\mathrm{A}}^{2}\left(\mathrm{P}\left(\mathscr{U}_{\mathrm{A}}\right), *_{\varepsilon}\right)
$$

defines an orientation for Bott inverted $\mathrm{P}\left(\mathcal{U}_{\mathrm{A}}\right)$. The corresponding A-equivariant formal group law is strictly multiplicative in the sense that its coproduct $\Delta$ has the property

$$
\Delta(\mathrm{x}(\varepsilon))=1 \otimes \mathrm{x}(\varepsilon)+\mathrm{x}(\varepsilon) \otimes 1-\beta \mathrm{x}(\varepsilon) \otimes \mathrm{x}(\varepsilon) .
$$

Here $\mathrm{x}(\varepsilon)$ is the coordinate of the formal group law.

Proof. The multiplication map on $\mathrm{P}\left(\mathscr{U}_{\mathrm{A}}\right)$ determined by tensor products of line bundles induces a map

$$
\begin{aligned}
\left(\Sigma^{\infty} \mathrm{P}\left(\mathscr{U}_{\mathrm{A}}\right)_{+}\left[\beta^{-1}\right]\right)_{\mathrm{A}}^{*}\left(\mathrm{P}\left(\mathscr{U}_{\mathrm{A}}\right)\right) & \\
\longrightarrow & \left(\Sigma^{\infty} \mathrm{P}\left(\mathscr{U}_{\mathrm{A}}\right)_{+}\left[\beta^{-1}\right]\right)_{\mathrm{A}}^{*}\left(\mathrm{P}\left(\mathscr{U}_{\mathrm{A}}\right) \times \mathrm{P}\left(\mathscr{U}_{\mathrm{A}}\right)\right) .
\end{aligned}
$$

In cohomology $\beta$ maps to $\beta_{1} \beta_{2}$, where $\beta_{i}$ is the image of $\beta$ under the map induced by the projection $\mathrm{pr}_{i}: \mathrm{P}\left(\mathscr{U}_{\mathrm{A}}\right) \times \mathrm{P}\left(\mathscr{U}_{\mathrm{A}}\right) \rightarrow \mathrm{P}\left(\mathscr{U}_{\mathrm{A}}\right)$ on the $i$ th factor. It follows that the coproduct of the corresponding $A$-equivariant formal group law induced by (1) is strictly multiplicative [4, Corollary 9.7], cf. [10, Lemma 3.2]. For the analogous example of A-equivariant unitary topological $K$-theory, see also $[6, \S 4]$.

REMARK 3.3. With these definitions the map

$$
\left(\Sigma^{\infty} \mathrm{P}\left(\mathscr{U}_{\mathrm{A}}\right)_{+}\left[\beta^{-1}\right]\right)_{\mathrm{A}}^{*}(-) \longrightarrow \mathrm{KU}_{\mathrm{A}}^{*}(-)
$$

is orientation preserving.

\section{Proof of the main result}

Proof of Theorem i.I. Lemma 3.2 implies there exists a unique map between A-equivariant oriented cohomology theories

$$
\mathrm{MU}_{\mathrm{A}}^{*}(-) \longrightarrow\left(\Sigma^{\infty} \mathrm{P}\left(\mathscr{U}_{\mathrm{A}}\right)_{+}\left[\beta^{-1}\right]\right)_{\mathrm{A}}^{*}(-)
$$

By reference to the universal property of $\mathrm{KU}_{\mathrm{A}}^{*}$ with respect to A-equivariant strictly multiplicative formal group laws in [5] there exists a unique $\mathrm{MU}_{\mathrm{A}^{-}}$ algebra map between A-equivariant coefficient rings

$$
\mathrm{KU}_{\mathrm{A}}^{*} \longrightarrow\left(\Sigma^{\infty} \mathrm{P}\left(\mathcal{U}_{\mathrm{A}}\right)_{+}\left[\beta^{-1}\right]\right)_{\mathrm{A}}^{*} \text {. }
$$


Combining the above with the A-equivariant Conner-Floyd theorem, cf. [2], [8], yields a canonical map between A-equivariant oriented cohomology theories

$$
\mathrm{KU}_{\mathrm{A}}^{*}(-)=\mathrm{MU}_{\mathrm{A}}^{*}(-) \otimes_{\mathrm{MU}_{\mathrm{A}}^{*}} \operatorname{Rep}_{\mathrm{U}}(\mathrm{A})\left[\beta, \beta^{-1}\right] \longrightarrow\left(\Sigma^{\infty} \mathrm{P}\left(\mathscr{U}_{\mathrm{A}}\right)_{+}\left[\beta^{-1}\right]\right)_{\mathrm{A}}^{*}(-) .
$$

By the universal property of $\mathrm{KU}_{\mathrm{A}}^{*}(-)$ the composite map

$$
\mathrm{KU}_{\mathrm{A}}^{*}(-) \longrightarrow\left(\Sigma^{\infty} \mathrm{P}\left(\mathscr{U}_{\mathrm{A}}\right)_{+}\left[\beta^{-1}\right]\right)_{\mathrm{A}}^{*}(-) \longrightarrow \mathrm{KU}_{\mathrm{A}}^{*}(-)
$$

is the identity.

Denote also by $\beta^{-1}\left(1-\xi_{\infty}\right)$ the map

$$
\left(\Sigma^{-2} \Sigma^{\infty} \mathrm{P}\left(\mathscr{U}_{\mathrm{A}}\right)_{+}\right)_{\mathrm{A}}^{*}(-) \longrightarrow\left(\Sigma^{\infty} \mathrm{P}\left(\mathscr{U}_{\mathrm{A}}\right)_{+}\left[\beta^{-1}\right]\right)_{\mathrm{A}}^{*}(-)
$$

corresponding to the orientation class for Bott inverted $\mathrm{P}\left(\mathscr{U}_{\mathrm{A}}\right)$, and likewise for

$$
\xi_{\infty}:\left(\Sigma^{\infty} \mathrm{P}\left(\mathscr{U}_{\mathrm{A}}\right)_{+}\right)_{\mathrm{A}}^{*}(-) \longrightarrow\left(\Sigma^{\infty} \mathrm{P}\left(\mathscr{U}_{\mathrm{A}}\right)_{+}\left[\beta^{-1}\right]\right)_{\mathrm{A}}^{*}(-) .
$$

The $\mathrm{KU}_{\mathrm{A}}^{*}(-)$-module structure on $\left(\Sigma^{\infty} \mathrm{P}\left(\mathscr{U}_{\mathrm{A}}\right)_{+}\left[\beta^{-1}\right]\right)_{\mathrm{A}}^{*}(-)$ implies the equality

$$
\xi_{\infty}=1-\beta\left(\beta^{-1}\left(1-\xi_{\infty}\right)\right) .
$$

Now consider the orientation preserving composite map of $\mathrm{KU}_{\mathrm{A}}^{*}(-)$-modules

$$
v:\left(\Sigma^{\infty} \mathrm{P}\left(\mathscr{U}_{\mathrm{A}}\right)_{+}\left[\beta^{-1}\right]\right)_{\mathrm{A}}^{*}(-) \longrightarrow \mathrm{KU}_{\mathrm{A}}^{*}(-) \longrightarrow\left(\Sigma^{\infty} \mathrm{P}\left(\mathscr{U}_{\mathrm{A}}\right)_{+}\left[\beta^{-1}\right]\right)_{\mathrm{A}}^{*}(-) .
$$

We have $v \circ\left(\beta^{-1}\left(1-\xi_{\infty}\right)\right)=\left(\beta^{-1}\left(1-\xi_{\infty}\right)\right)$ and $v \circ \beta\left(\beta^{-1}\left(1-\xi_{\infty}\right)\right)=$ $\beta\left(\beta^{-1}\left(1-\xi_{\infty}\right)\right)$. Hence $v \circ \xi_{\infty}=\xi_{\infty}$. From the universal property of $\left(\Sigma^{\infty} \mathrm{P}\left(\mathscr{U}_{\mathrm{A}}\right)_{+}\left[\beta^{-1}\right]\right)_{\mathrm{A}}^{*}(-)$ it follows that $v$ is the identity map.

To summarize, the above shows there exists an isomorphism of A-equivariant oriented cohomology theories

$$
\left(\Sigma^{\infty} \mathrm{P}\left(\mathscr{U}_{\mathrm{A}}\right)_{+}\left[\beta^{-1}\right]\right)_{\mathrm{A}}^{*}(-) \stackrel{\cong}{\longrightarrow} \mathrm{KU}_{\mathrm{A}}^{*}(-) .
$$

It remains to show that (2) lifts, uniquely up to isomorphisms, to an isomorphism between oriented ring spectra in the equivariant homotopy category. This follows by observing that there exist no nontrivial phantom maps

$$
\left(\Sigma^{\infty} \mathrm{P}\left(\mathscr{U}_{\mathrm{A}}\right)_{+}\left[\beta^{-1}\right]\right)^{\wedge n} \longrightarrow \mathrm{KU}_{\mathrm{A}} .
$$

To wit, identify $\left(\Sigma^{\infty} \mathrm{P}\left(\mathscr{U}_{\mathrm{A}}\right)_{+}\left[\beta^{-1}\right]\right)^{\wedge n}$ with the homotopy colimit

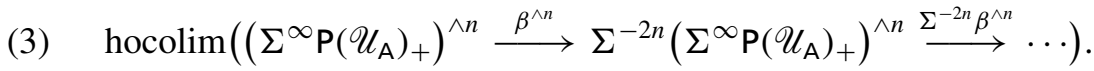


Applying $\mathrm{KU}_{\mathrm{A}}^{*}(-)$ to (3) yields a sequence whose $\lim ^{1}$-term vanishes because the group $\operatorname{KU}_{\mathrm{A}}^{*}\left(\mathrm{P}\left(\mathscr{U}_{\mathrm{A}}\right)\right)$ is trivial in odd degrees. Combining Cole's computation of the $\mathrm{KU}_{\mathrm{A}}^{*}$-cohomology of $\mathrm{P}\left(\mathscr{U}_{\mathrm{A}}\right)\left[1\right.$, Theorem 4.3] and the fact that $\mathrm{KU}_{\mathrm{A}}^{1}$ is the trivial group finishes the proof.

Remark 4.1. The proof given in [10] of the (non-equivariant) motivic homotopy theoretic analog of our main result incited and predates the work presented here. The reader might want to compare the current results to those of Gepner and Snaith [3] and also of Naumann, Spitzweck and Østvær [7].

Remark 4.2. We wish to thank the referee for helpful comments that enabled us to improve this paper. The referee also pointed out that our main result cannot be generalized to non-abelian groups, cf. [9, Remark 3.4], and suggested that, for finite abelian groups, an induction argument might lead to an alternate proof of our main result.

\section{REFERENCES}

1. Cole, M., Greenlees, J. P. C., and Kriz, I., The universality of equivariant complex bordism, Math. Z. 239 (2002), 455-475.

2. Costenoble, S. R., The equivariant Conner-Floyd isomorphism, Trans. Amer. Math. Soc. 304 (1987), 801-818.

3. Gepner, D., and Snaith, V., On the motivic spectra representing algebraic cobordism and algebraic K-theory, Documenta Math. 14 (2009), 359-396.

4. Greenlees, J. P. C., Equivariant formal group laws and complex oriented cohomology theories, Equivariant stable homotopy theory and related areas (Stanford, CA 2000), Homology Homotopy Appl. 3 (2001), 225-263.

5. Greenlees, J. P. C., Multiplicative equivariant formal group laws, J. Pure Appl. Algebra 165 (2001), 183-200.

6. Greenlees, J. P. C., Equivariant versions of real and complex connective $K$-theory, Homology, Homotopy Appl. 7 (2005), 63-82.

7. Naumann, N., Spitzweck, M., and Østvær, P. A., Chern classes, K-theory and Landweber exactness over nonregular base schemes, pp. 307-317 in: Motives and Algebraic Cycles, Fields Inst. Commun. 56, Amer. Math. Soc., Providence, RI, 2009.

8. Okonek, C., Der Conner-Floyd-Isomorphismus für Abelsche Gruppen, Math. Z. 179 (1982), 201-212.

9. Snaith, V., A model for equivariant K-theory, C. R. Math. Rep. Acad. Sci. Canada 3 (1981), $143-147$.

10. Spitzweck, M., and Østvær, P. A., The Bott inverted infinite projective space is homotopy algebraic K-theory, Bull. London Math. Soc. 41 (2009), 281-292.

FAKULTÄT FÜR MATHEMATIK

UNIVERSITÄT REGENSBURG

GERMANY

E-mail: Markus.Spitzweck@mathematik.uni-regensburg.de
DEPARTMENT OF MATHEMATICS

UNIVERSITY OF OSLO

NORWAY

E-mail: paularne@math.uio.no 